\title{
TEMPERATURE SENSITIVITY OF SOLID-WAVE GYROSCOPES
}

Erdal Yilmaz ${ }^{1}$ and David Bindel ${ }^{2}$

${ }^{1}$ Applied Physics, Cornell University, Ithaca, New York, USA

${ }^{2}$ Department of Computer Science, Cornell University, Ithaca, New York, USA

\section{ABSTRACT}

We analyze the change of angular gain and vibration frequency of solid-wave gyroscopes as a result of geometry perturbations due to thermal expansion. We formulate a temperature sensitivity analysis by assuming a linear dependence of material properties to temperature, and quantify it for common device geometries.

\section{INTRODUCTION}

The lumped model of a solid-wave gyroscope involves two degenerate, coupled, damped and driven harmonic oscillators:

$\ddot{\mathbf{q}}+2\left(\gamma \mathbf{I}+A_{g} \Omega \mathbf{J}\right) \dot{\mathbf{q}}+\omega^{2} \mathbf{q}=\mathbf{f}, \mathbf{I} \equiv\left[\begin{array}{ll}1 & 0 \\ 0 & 1\end{array}\right], \mathbf{J} \equiv\left[\begin{array}{rr}0 & -1 \\ 1 & 0\end{array}\right]$

where the vectors $\mathbf{q}(t)$ and $\mathbf{f}(t)$ correspond to generalized coordinates and drive forces respectively. $\omega$ is the undamped angular vibration frequency and $\Omega$ is the rotation rate of the gyro platform. $\gamma=\omega / 2 Q$ is the damping coefficient and $A_{g}$ is known as angular gain [1].

As the device temperature $T$ changes, the coefficients of the lumped model drift. Computing $\gamma(T), A_{g}(T)$ and $\omega(T)$ requires modeling geometric changes of the resonator. Since the damping coefficient $\gamma(T)$ depends on the relevant damping mechanisms, we only compute sensitivity of $A_{g}(T)$ and $\omega(T)$.

\section{METHOD}

Typical solid-wave gyroscope resonators are axisymmetric shells clamped at one of two boundaries and free on the other (Fig. 1). The cross section of the shell's midsurface forms a plane curve on $(r, z)$-plane. We parametrize this curve by its arclength $s$ and use $h, L$ and $d$ to represent the shell thickness, the length of the midline curve, and the distance from the clamped boundary to the symmetry axis, respectively.

Temperature variations change both material properties and geometry of resonators. The elastic coefficients of conventional materials used in microfabrication change almost linearly within the standard range of operational temperatures: $E(T)=E_{0}\left(1+\alpha_{E}\left(T-T_{0}\right)\right), \nu(T)=\nu_{0}(1+$ $\left.\alpha_{\nu}\left(T-T_{0}\right)\right)$. Also, due to thermal expansion, the device geometry and material density change slightly: $\rho(T)=$ $\rho_{0}\left(1-3 \alpha_{L}\left(T-T_{0}\right)\right)$, where $\alpha_{L}$ is the thermal coefficient of expansion of the resonator material. We assume the post, attached at $r=d$, expands in radial direction with thermal coefficient $\alpha_{S}$ corresponding to the substrate material.

First, we discretize the axisymmetric thermal expansion problem: $\mathbf{K}_{T} \mathbf{u}_{T}=\mathbf{f}_{T}$, which is inherently nonlinear; but we only need $\frac{d \mathbf{u}_{T}}{d T}=\mathbf{K}_{T}^{-1} \frac{d \mathbf{f}_{T}}{d T}$ in our analysis. We use this solution to differentiate the resonator shape with respect to
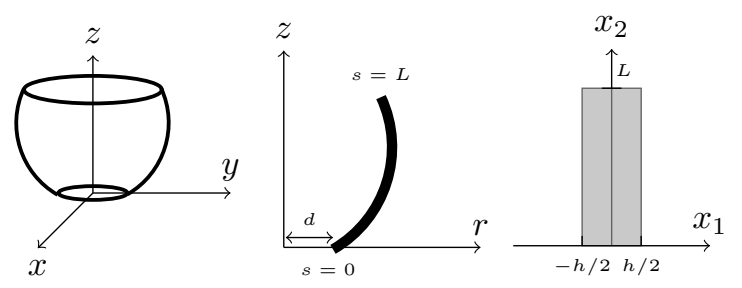

Fig. 1: Left: Axisymmetric shell. Clamped at the bottom boundary, free at the top boundary. Center: Cross section in $(r, z)$-plane. Right: Computational mapped domain.

temperature and solve for the sensitivities of the lumped model coefficients [2]. We non-dimensionalize the equations using units of length $l^{*}=L$, time $\tau^{*}=L / \sqrt{E_{0} / \rho_{0}}$, mass $m^{*}=\rho_{0} L^{3}$ and temperature $T^{*}=\alpha_{L}^{-1}$.

\section{RESULTS}

We investigated temperature sensitivities for three common geometries (Fig. 2). They all have the same midline length and shell thickness. We selected $d$ so that radial extents are the same. Table 1 shows the numerical values used in simulations. In Table 2, we tabulated sensitivities for $m=2$ and $m=3$ modes for each resonator.

Since all thermal coefficients are small, we can write temperature sensitivity of angular gain or frequency as a sum of all effects: $y=C_{L} \alpha_{L}+C_{S} \alpha_{S}+C_{E} \alpha_{E}+C_{\nu} \alpha_{\nu}$, where $y$ is either $\left(1 / A_{g}\right) d A_{g} / d T$ or $(1 / \omega) d \omega / d T$, and $C$ 's are constants for a particular resonator. By setting all $\alpha$ 's to zero except one, we can compute the contribution of each thermal effect (Table 3, 4).

Design $\mathcal{C}$ is particularly sensitive to Poisson's ratio, and so is more sensitive to temperature variations than $\mathcal{H}$ and $\mathcal{T}$. The effects of substrate thermal expansion matter just as much to thermal sensitivity in the other designs, highlighting the importance of selection of the post material and radius. For all devices and modes, angular gain is independent of Young's modulus. Frequency sensitivity depends strongly on thermal variations of Young's modulus, much more than on any geometric effects; hence, new geometries are unlikely to improve frequency sensitivity. In contrast, by proper choice of the post radius, we can achieve temperature insensitive angular gain (Fig. 3). We can also formulate basic shape optimization problems as in Fig. 4, where the cost function is defined proportional to $\left(\frac{1}{A_{g}} \frac{\dot{A}_{g}}{d T}\right)^{2}$.

\section{REFERENCES}

[1] V. Zhuravlev, "Theoretical foundations of solid-state wave gyroscopes," Mechanics of Solids, vol. 28, pp. 3-15, 1993.

[2] E. Yilmaz and D. Bindel, "Effects of imperfections on solid-wave gyroscope dynamics," in SENSORS, 2013 IEEE, Nov 2013, pp. 1-4.

Solid-State Sensors, Actuators and Microsystems Workshop Hilton Head Island, South Carolina, June 5-9, 2016 


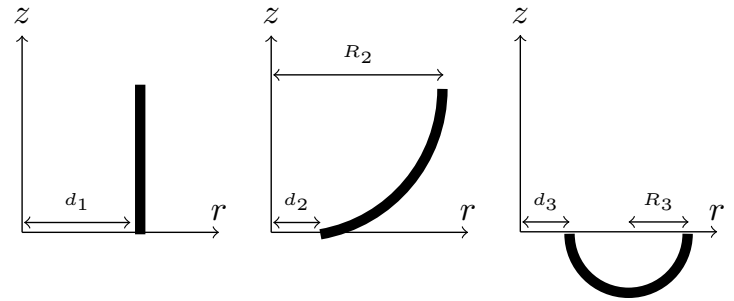

Fig. 2: Left: Cylindrical shell C. Center: Truncated hemispherical shell $\mathcal{H}$. Right: Half-toroidal shell $\mathcal{T}$.

Table 1: Material properties and geometry of resonators

\begin{tabular}{lll}
\hline \hline Parameter & Symbol & Value \\
\hline Young's modulus & $E_{0}$ & $80 \mathrm{GPa}$ \\
Poisson's ratio & $\nu_{0}$ & 0.26 \\
Material density & $\phi_{0}$ & $2200 \mathrm{~kg} / \mathrm{m}^{3}$ \\
Thermal expansion coefficient of resonator & $\alpha_{L}$ & $1.4 \times 10^{-6} \mathrm{~K}^{-1}$ \\
Thermal expansion coefficient of substrate & $\alpha_{S}$ & $2.6 \times 10^{-6} \mathrm{~K}^{-1}$ \\
Thermal coefficient of $E$ & $\alpha_{E}$ & $2.5 \times 10^{-4} \mathrm{~K}^{-1}$ \\
Thermal coefficient of $\nu$ & $\alpha_{\nu}$ & $3.0 \times 10^{-4} \mathrm{~K}^{-1}$ \\
Thickness to length ratio & $h / L$ & 0.01 \\
Post radius to length ratio (cylindrical) & $d_{1} / L$ & $12 / 5 \pi$ \\
Post radius to length ratio (spherical) & $d_{2} / L$ & $12 \sin (\pi / 12) / 5 \pi$ \\
Post radius to length ratio (toroidal) & $d_{3} / L$ & $2 / 5 \pi$ \\
Radius of hemisphere to length ratio & $R_{2} / L$ & $12 / 5 \pi$ \\
Minor radius of torus to length ratio & $R_{3} / L$ & $1 / \pi$ \\
\hline \hline
\end{tabular}

Table 2: Temperature sensitvities

\begin{tabular}{|c|c|c|c|c|c|}
\hline $\mathcal{D}$ & $m$ & $A_{g}$ & $\frac{1}{A_{g}} \frac{d A_{g}}{d T}\left(\frac{p p b}{K}\right)$ & $\omega\left(\frac{1}{L} \sqrt{\frac{E_{0}}{\rho_{0}}}\right)$ & $\frac{1}{\omega} \frac{d \omega}{d T}\left(\frac{p p m}{K}\right)$ \\
\hline $\mathcal{C}$ & 2 & 0.744 & -613 & 0.345 & 102.2 \\
& 3 & 0.577 & -1694 & 0.214 & 108.5 \\
\hline $\mathcal{H}$ & 2 & 0.554 & -305 & 0.028 & 108.2 \\
& 3 & 0.487 & -126 & 0.061 & 101.5 \\
\hline $\mathcal{T}$ & 2 & 0.304 & -576 & 0.040 & 121.6 \\
& 3 & 0.357 & -220 & 0.091 & 110.5 \\
\hline
\end{tabular}

Table 3: Thermal components of angular gain sensitivity

\begin{tabular}{|c|c|c|c|c|c|c|}
\hline $\mathcal{D}$ & $m$ & $\frac{1}{A_{g}} \frac{d A_{g}}{d T}\left(\frac{p p b}{K}\right)$ & $C_{L} \alpha_{L}$ & $C_{S} \alpha_{S}$ & $C_{E} \alpha_{E}$ & $C_{\nu} \alpha_{\nu}$ \\
\hline $\mathcal{C}$ & 2 & -613 & 56 & -313 & 0 & -356 \\
& 3 & -1694 & 251 & -199 & 0 & -1747 \\
\hline $\mathcal{H}$ & 2 & -305 & -105 & -269 & 0 & 70 \\
& 3 & -126 & 0 & -145 & 0 & 18 \\
\hline $\mathcal{T}$ & 2 & -576 & -4 & -441 & 0 & -131 \\
& 3 & -220 & 0 & -231 & 0 & 11 \\
\hline
\end{tabular}

Table 4: Thermal components of frequency sensitivity

\begin{tabular}{|c|c|c|c|c|c|c|}
\hline $\mathcal{D}$ & $m$ & $\frac{1}{\omega} \frac{d \omega}{d T}\left(\frac{p p m}{K}\right)$ & $C_{L} \alpha_{L}$ & $C_{S} \alpha_{S}$ & $C_{E} \alpha_{E}$ & $C_{\nu} \alpha_{\nu}$ \\
\hline $\mathcal{C}$ & 2 & 102.16 & -4.90 & 0.67 & 125 & -18.6 \\
& 3 & 108.48 & -7.96 & 1.05 & 125 & -9.5 \\
\hline $\mathcal{H}$ & 2 & 108.25 & -6.73 & 0.37 & 125 & -10.3 \\
& 3 & 101.55 & 0.64 & -1.06 & 125 & -23.0 \\
\hline $\mathcal{T}$ & 2 & 121.63 & 0.64 & -0.28 & 125 & -3.7 \\
& 3 & 110.52 & 0.70 & -0.60 & 125 & -14.6 \\
\hline
\end{tabular}

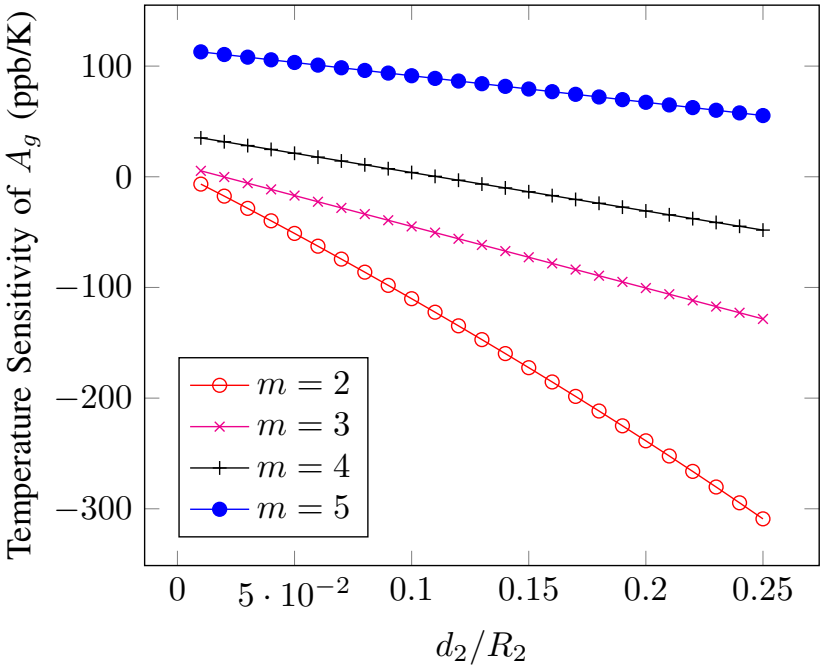

Fig. 3: Temperature sensitivities of angular gain of truncated hemispherical shell as a function of post radius to shell radius ratio. Smaller posts are desirable for $m=2,3$ modes. It seems possible to design $m=4$ with $A_{g}$ insensitive to temperature.

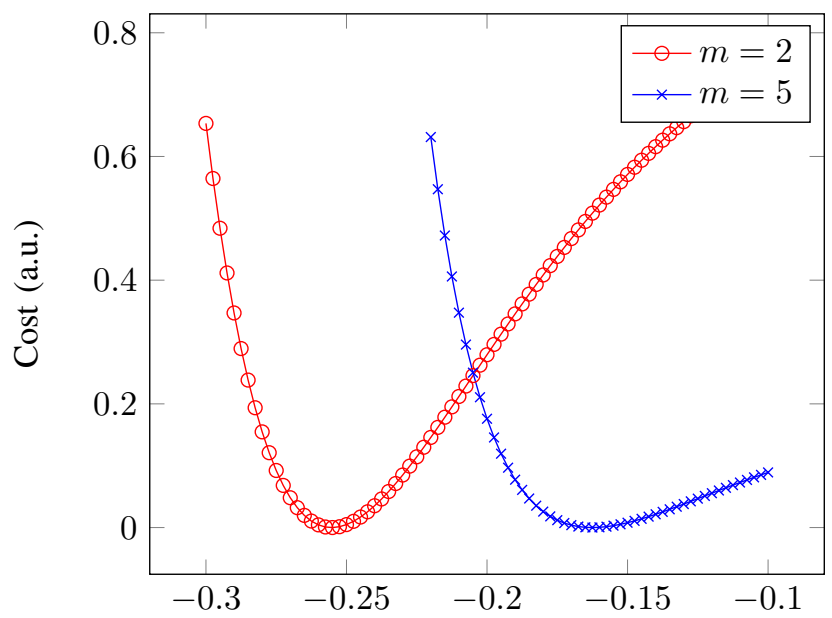

Coordinate along the dashed line

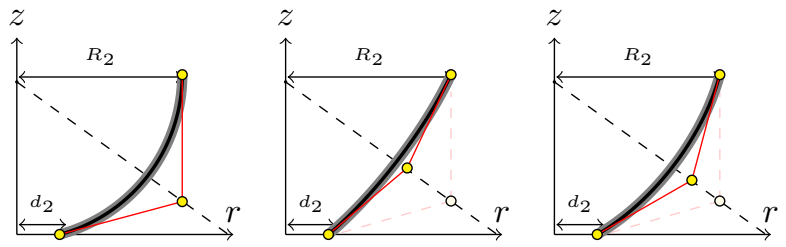

Fig. 4: The simplest shape optimization problem. Bottom left: Three control points representing truncated hemispherical shell. The middle point is free to move along the dashed line while the other two are fixed. Bottom middle and right: Optimal shape and new position of the control point which minimizes temperature sensitivity of angular gain for modes $m=2$ and $m=5$ respectively. Top: Local minimum of the cost function for $m=2$ and $m=5$ by sweeping the position of control point on the dashed line. 